\title{
Mathematical energy minimization model for joining boron nitride fullerene with several BN nanostructures
}

\author{
Nawa A. Alshammari ${ }^{1}$ (D) \\ Received: 23 February 2021 / Accepted: 28 July 2021 / Published online: 11 August 2021 \\ (c) The Author(s) 2021
}

\begin{abstract}
Nanoscale materials have gained considerable interest because of their special properties and wide range of applications. Many types of boron nitride at the nanoscale have been realized, including nanotubes, nanocones, fullerenes, tori, and graphene sheets. The connection of these structures at the nanoscale leads to merged structures that have enhanced features and applications. Modeling the joining between nanostructures has been adopted by different methods. Namely, carbon nanostructures have been joined by minimizing the elastic energy in symmetric configurations. In other words, the only considerable curvature in the elastic energy is the axial curvature. Accordingly, because it has nanoscale structures similar to those in carbon, BN can also be joined and connected by using this method. On the other hand, different methods have been proposed to consider the rotational curvature because it has a similar size. Based on that argument, the Willmore energy, which depends on both curvatures, has been minimized to join carbon nanostructures. This energy is used to identify the joining region, especially for a three-dimensional structure. In this paper, we expand the use of Willmore energy to cover the joining of boron nitride nanostructures. Therefore, because catenoids are absolute minimizers of this energy, pieces of catenoids can be used to connect nanostructures. In particular, we joined boron nitride fullerene to three other BN nanostructures: nanotube, fullerene, and torus. For now, there are no experimental or simulation data for comparison with the theoretical connecting structures predicted by this study, which is some justification for the suggested simple model shown in this research. Ultimately, various nanoscale BN structures might be connected by considering the same method, which may be considered in future work.
\end{abstract}

Keywords $\mathrm{BN}$ nanotubes $\cdot \mathrm{BN}$ fullerene $\cdot \mathrm{BN}$ torus $\cdot$ Willmore energy $\cdot$ Calculus of variations

\section{Introduction}

Nanoscale materials have considerable interest because of their extraordinary properties and applications. In particular, carbon structures at the nanoscale such as nanotubes, nanocones, graphene sheets, tori, and fullerenes have received significant interest for application in a variety of fields, including microelectronics, sensing and actuation systems, biotechnologies, composite materials, and energy storage [12]. On the other hand, boron nitride (BN) structures at the nanoscale are distinctive because of their similarity to carbon structures at the nanoscale. Because

Nawa A. Alshammari

N.Alshammari@seu.edu.sa

1 Department of Mathematics, College of Science and Theoretical Studies, Saudi Electronic University, Riyadh, Saudi Arabia of their remarkable properties, these particular structures have broad applications in improving nanodevices [1, 27]. The hexagonal BN structure contains boron and nitrogen atoms bound by strong covalent bonds. In addition, these nanostructures can be found in many forms, such as $\mathrm{BN}$ fullerene (BN fullerene), BN nanotorus (BNNTR), $\mathrm{BN}$ nanotube (BNNT), BN nanocone (BNNC), and BN graphene (BN graphene) forms [18, 25].

Similar to carbon nanostructures, these nanostructures have favorable thermal and mechanical properties such as high thermal conductivity, low density, high tensile strength, and membrane stiffness. Additionally, their atomic composition provides them with characteristics surpassing those of carbon nanostructures, such as stronger resistance to oxidation and chemical stability at high temperatures [12, 14, 17, 20, 23]. Furthermore, BN nanostructures have outstanding applications in energy storage, optoelectronics, electronics, biomedical medicine, and nanosemiconductor devices [13]. 
A BNNT can be formed as a tube structure of a hexagonal lattice involving organized boron and nitrogen atoms, and this structure was first discovered in 1994. BNNTs have an important interest among researchers because of their unique characteristics. In particular, BNNTs are electrically insulating with a band gap of $6 \mathrm{eV}$, and they are stable up to $800{ }^{\circ} \mathrm{C}$ in air. BNNTs can be considered to have outstanding thermal conductivities for high Young's moduli above 1.3 TPa and superhydrophobicity. BNNTs are also piezoelectric and useful for spintronic devices [8].

In 1985 , after the discovery of fullerene $\mathrm{C}_{60}$, researchers attempted to find fullerene-like compounds comprising other elements. Boron nitride fullerenes are the most attractive structures, such as $\mathrm{B}_{36} \mathrm{~N}_{36}$, which is quite similar to carbon fullerene $\mathrm{C}_{60}$. $\mathrm{BN}$ fullerenes have attracted much scientific research. That is, they have a higher heat resistance and a wider band gap in air than carbon structures. $\mathrm{BN}$ fullerenes are useful in many aspects, for example, in electronic devices, insulator lubricants, and high semiconductors. BN fullerenes that include lightweight structures are capable of storing many gas molecules per unit weight. Additionally, they are useful in gas storage, molecular sieves, or nanomembranes [16].

A boron nitride nanotorus (BNNTR) can be defined as a $\mathrm{BN}$ nanotube bent such that its ends are connecting together. This nanostructure has been studied in many studies based on its structure and unique properties. Different applications have been found in the literature, such as in ultrafast optical filters and nanoantennas sensitive to high-frequency electromagnetic signals $[18,30]$.

Connecting or joining nanostructures to other nanostructures has advantages because it leads to new nanostructures with enhanced physiochemical and electrochemical performance, affording them a broad range of uses in various fields [10, 19, 28, 32]. New joined nanoscale structures might develop the physiochemical and electrochemical performance of joined nanoscale structures, such as in nanosensors and nanooscillators. In particular, the newly joined nanoscale structures are helpful for energy storage, for designing probes for scanning tunneling microscopy, and as carriers for drug delivery [27]. Therefore, many methods have been used to join and connect nanomaterials. For example, elastic energy is employed to join different carbon nanostructures, as these nanoscale structures are supposed to deform with perfect elasticity. In particular, by minimizing the squared curvature, the EulerLagrange equation is obtained, which is applied to define the joint region between carbon nanomaterials. As a result of this method, many carbon nanostructures have been connected, such as carbon nanotubes and graphene, nanotubes and fullerenes, nanotubes and nanocones, fullerenes and graphenes as two fullerenes, two nanocones, nanocones and fullerenes, nanocones and fullerenes, and two parallel sheets of graphene, as detailed in [9, 11, 24] and [2]. Furthermore, another method was utilized to connect carbon nanostructures, that is, the Willmore energy [29]. This method supports the argument that considers the rotational curvature because it has a similar size. As a natural generalization of elastic energy, the Willmore energy, which involves axial curvature and rotational curvature, is used to determine the surface connection of two carbon nanoscale structures. As a result of this energy, carbon nanostructures have been joined and connected, for example, carbon nanotubes to fullerenes and two fullerenes to each other, as detailed in [29]. Furthermore, another study used this energy to join a carbon nanotorus and a nanotube [30]. In addition, similar techniques have been utilized and investigated by other researchers, for example, in [22] and [21].

On the other hand, because of their similarity to carbon in nanoscale structures, $\mathrm{BN}$ in nanoscale structures has been joined and connected using elastic energy. In detail, other studies have used elastic energy to connect and join $\mathrm{BN}$ nanostructures: $\mathrm{BN}$ nanocones to nanotubes and $\mathrm{BN}$ graphene to other $\mathrm{BN}$ nanostructures; and nanotubes to $\mathrm{BN}$ nanocones, as shown in [3] and [4], respectively. In this paper, the Willmore energy method is utilized to join and connect BN nanostructures.

The Willmore energy can be written as:

$W=\int_{M} H^{2} d \mu$

where $H$ denotes the mean curvature (which involves the sum of the rotational curvature and the axial curvature) of surface $M$ and $d \mu$ is the area element [31]. The Willmore energy has been demonstrated to have possible applications in many different aspects of molecular, biological, and nanotechnology science. On the other hand, catenoids, as absolute minimizers of the Willmore energy, have numerous uses in nanomagnetism [5-7, 15, 26]. This paper expands the method of using catenoids to establish the conformation of the connection of $\mathrm{BN}$ nanostructures, namely, a BN nanotube to a $\mathrm{BN}$ fullerene and two $\mathrm{BN}$ fullerenes to each other.

In this research, we recognize that the existence of this predicted structure has not been achieved experimentally. The purpose of this research is to accommodate the major features that encapsulate the dominant physical effects so that we might imagine a real physical system in terms of departures from an ideal model. In particular, this research modeled only the mathematics of the energy inherent in the curved surface, which can be thought of as a result of chemical bonding. This model does not take into account chemical issues, such as the position of nanostructure atoms and bonds. However, the curved profiles of the graphene fold obtained by minimizing the elastic energy 
using calculus of variations are in excellent agreement with the experimental results [9].

In the following section, the essential equations to model the joining region between $\mathrm{BN}$ nanostructures are determined. In "Results", the results are provided in three subsections, where the connection of a BN fullerene to a BN nanotube is given in "Joining BN nanotubes and fullerenes", between two fullerenes is specified in "Joining two BN fullerenes", and between a fullerene and torus is provided in "Joining a BN fullerene and BN nanotorus". Finally, "Conclusions" provides the conclusion of this paper.

\section{Model}

We may write the mean curvature in terms of the axial and rotational curvatures $\kappa_{a}$ and $\kappa_{r}$, respectively, which is called the Willmore energy function, as follows:

$$
J[y]=\int\left(\kappa_{a}+\kappa_{r}\right)^{2} d \mu+\lambda \int d \mu,
$$

where $d \mu$ is an area element, $\lambda$ is a Lagrange multiplier corresponding to an area constraint, and $H$ denotes the sum of both curvatures, which is the mean curvature. Assume that the joining will have a catenoid surface $S=\{(x, y, z)$ : $x=r \cos \theta, y=r \sin \theta, z=f(r)\}$, as shown in Fig. 1 . The mean curvature can be written in terms of both curvatures as

$$
H=\kappa_{a}+\kappa_{r}=-\frac{f^{\prime \prime}(r)}{\left(1+f^{\prime 2}(r)\right)^{\frac{3}{2}}}-\frac{f^{\prime}(r)}{r \sqrt{f^{\prime 2}(r)+1}},
$$

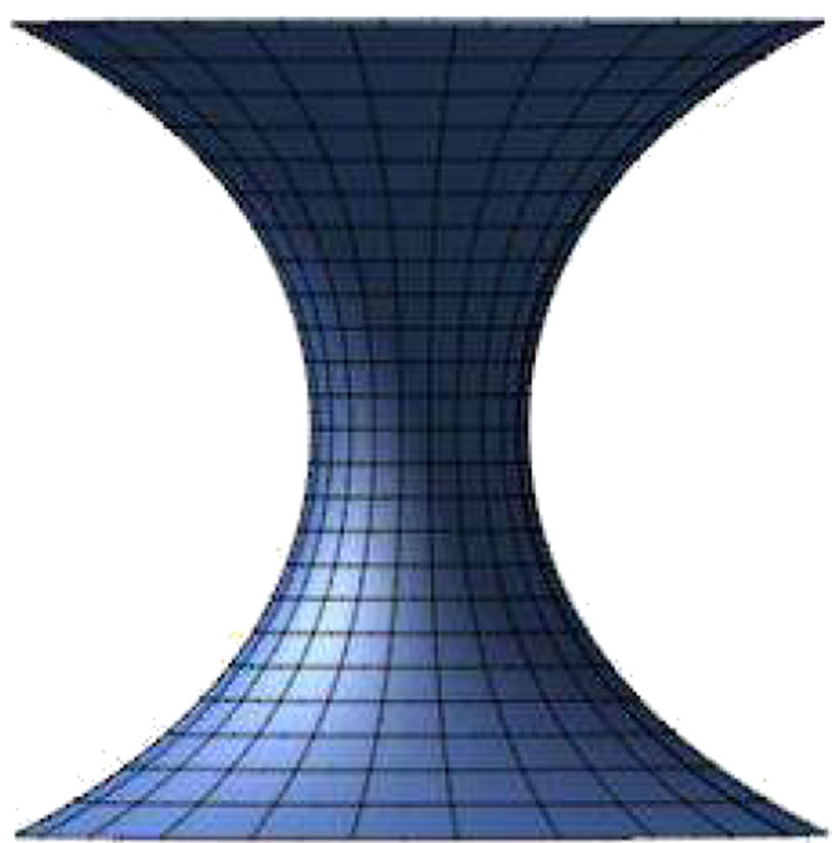

Fig. 1 Catenoid surface if $H=0$, this gives an absolute minimizer of the Willmore energy, the general solution might be expressed as

$$
f(r)= \pm \frac{\ln \left(a r+\sqrt{a^{2} r^{2}-1}\right)}{a}+b,
$$

where $a$ and $b$ are arbitrary constants. In addition, this solution can be written as:

$$
f(r)= \pm \frac{\cosh ^{-1}(a r)}{a}+b .
$$

This demonstrates the joining surface, as a catenoid, between two nanostructures. For more details of derivation, we refer the reader to [29].

In this paper, rotationally symmetric boron nitride nanostructures are joined and connected by using a part of the catenoid. In particular, we employ the boundary conditions that we have when matching the gradient at the connection points with the coordinates to find the arbitrary constants. In the following section, a catenoid is used to construct new $\mathrm{BN}$ nanostructures resulting from the connection between two different BN nanostructures: first, a fullerene with a nanotube; second, two fullerenes; and finally, a fullerene with a torus.

\section{Results}

\section{Joining BN nanotubes and fullerenes}

Figure 2 presents a three-dimensional schematic of the joined structure, while a part of the catenoid is used to join a boron nitride nanotube and a fullerene. Here, the curve of the catenoid can be written as

$z=-\frac{\cosh ^{-1}(a r)}{a}+b$

with constants $a$ and $b$. Assume that the radius of the $\mathrm{BN}$ nanotube is the specified value $r=r_{t}$. Furthermore, the fullerene equation can be expressed as

$z=A+\sqrt{R^{2}-r^{2}}$

when the radius of the fullerene is $R$ and $A$ denotes the constant specifying the fullerene on the negative $z$-axis, which can be specified by the position of the connection to the catenoid. Moreover, assume that the nanotube connects the catenoid at $(r, z)=\left(r_{t}, 0\right)$ and that the other side of the catenoid connects the fullerene at $(r, z)=\left(r_{f}, z_{f}\right)$. Additionally, $a, b, A$, and $r_{f}$ are constants to be determined, where $z_{f}$ might be known as long as we know $r_{f}$. At the connection points and from the boundary condition, the coordinates should be consistent with the gradient. Therefore, on $\left(r_{t}, 0\right)$, using Eq. 1, we obtain

$b=\frac{\cosh ^{-1}\left(a r_{t}\right)}{a}$, 


\section{BN nanotube}

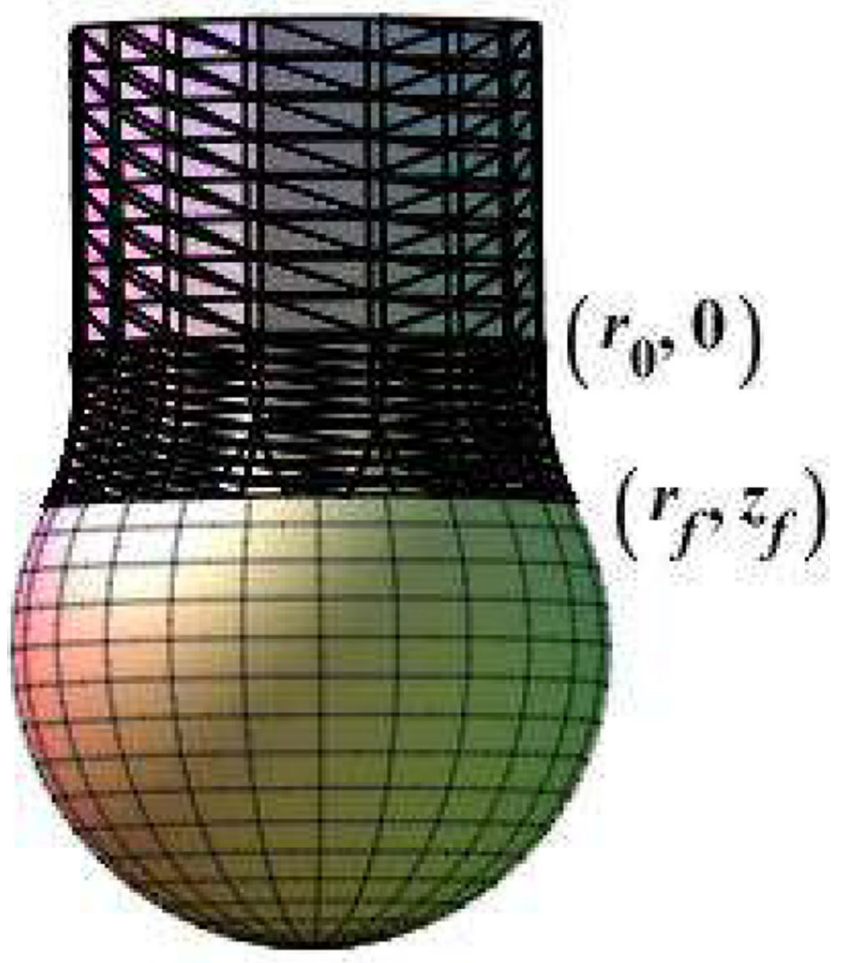

\section{BN Fullerene}

Fig. 2 A part of the catenoid joins between the BN nanotube and BN fullerene

and from Eq. 1, we have

$\frac{d z}{d r}=-\frac{1}{\sqrt{a^{2} r^{2}-1}}$.

The gradient at $\left(r_{t}, 0\right)$ gives

$-\frac{1}{\sqrt{a^{2} r_{t}^{2}-1}}=\infty$,

that is,

$a=\frac{1}{r_{t}}$,

and $b=0$.

Additionally, on $\left(r_{f}, z_{f}\right)$,

$-\frac{\cosh ^{-1}\left(a r_{f}\right)}{a}=A+\sqrt{R^{2}-r_{f}^{2}}$,

which gives

$A=-r_{t} \cosh ^{-1}\left(r_{f} / r_{t}\right)-\sqrt{R^{2}-r_{f}^{2}}$, matching the gradient results

$-\frac{1}{\sqrt{a^{2} r_{f}^{2}-1}}=\frac{r_{f}}{\sqrt{R^{2}-r_{f}^{2}}}$,

and from Eq. 3, we obtain

$r_{f}=\sqrt{R r_{t}}$,

and

$A=-r_{t} \cosh ^{-1} \sqrt{\frac{R}{r_{t}}}-\sqrt{R\left(R-r_{t}\right)}$.

Substituting Eqs. 3, 4, and 5 to Eqs. 1 and 2, with particular values for $R$ and $r_{t}$, we obtain the connection shape between the BN nanotube and fullerene by using the catenoid curve, as shown in Fig. 3

\section{Joining two BN fullerenes}

Here, the case of joining two fullerenes is considered (see Fig. 4). If we suppose the catenoid curve to be

$z= \pm \frac{\cosh ^{-1}(a r)}{a}+b$,

with constants $a$ and $b$, then the positive sign represents the upper part of the catenoid, and the negative sign represents the lower part of the catenoid. As at the point $\left(r_{t}, 0\right)$ the gradient is $\infty$, we obtain $\frac{1}{\sqrt{a^{2} r_{t}^{2}-1}}=\infty$, that is,

$a=\frac{1}{r_{t}}, \quad b=0$.

Now, we assume that the upper fullerene equation is:

$z=A_{1}-\sqrt{R_{1}^{2}-r^{2}}$,

while the radius of the upper fullerene is denoted by $R_{1}$, and $A_{1}$ is the constant that defines the center of the fullerene on the positive $z$-axis. Additionally, the lower fullerene can be written as

$z=A_{2}+\sqrt{R_{2}^{2}-r^{2}}$

where $R_{2}$ denotes the radius of the lower fullerene, and $A_{2}$ denotes the constant that defined the center of the fullerene with the negative $z$-axis. Afterwards, at $\left(r_{f_{1}}, z_{f_{1}}\right)$ and from Eqs. 6 and 8, we obtain

$A_{1}=r_{t} \cosh ^{-1}\left(r_{f_{1}} / r_{t}\right)+\sqrt{R_{1}^{2}-r_{f_{1}}^{2}}$.

Furthermore, at point $\left(r_{f_{2}}, z_{f_{2}}\right)$, while the lower fullerene is connected to the lower catenoid, we have

$A_{2}=-r_{t} \cosh ^{-1}\left(r_{f_{2}} / r_{t}\right)-\sqrt{R_{2}^{2}-r_{f_{2}}^{2}}$.

Next, after matching the gradients at points $\left(r_{f_{1}}, z_{f_{1}}\right)$ and $\left(r_{f_{2}}, z_{f_{2}}\right)$, we have

$r_{f_{1}}=\sqrt{R_{1} r_{t}}$,

$r_{f_{2}}=\sqrt{R_{2} r_{t}}$. 


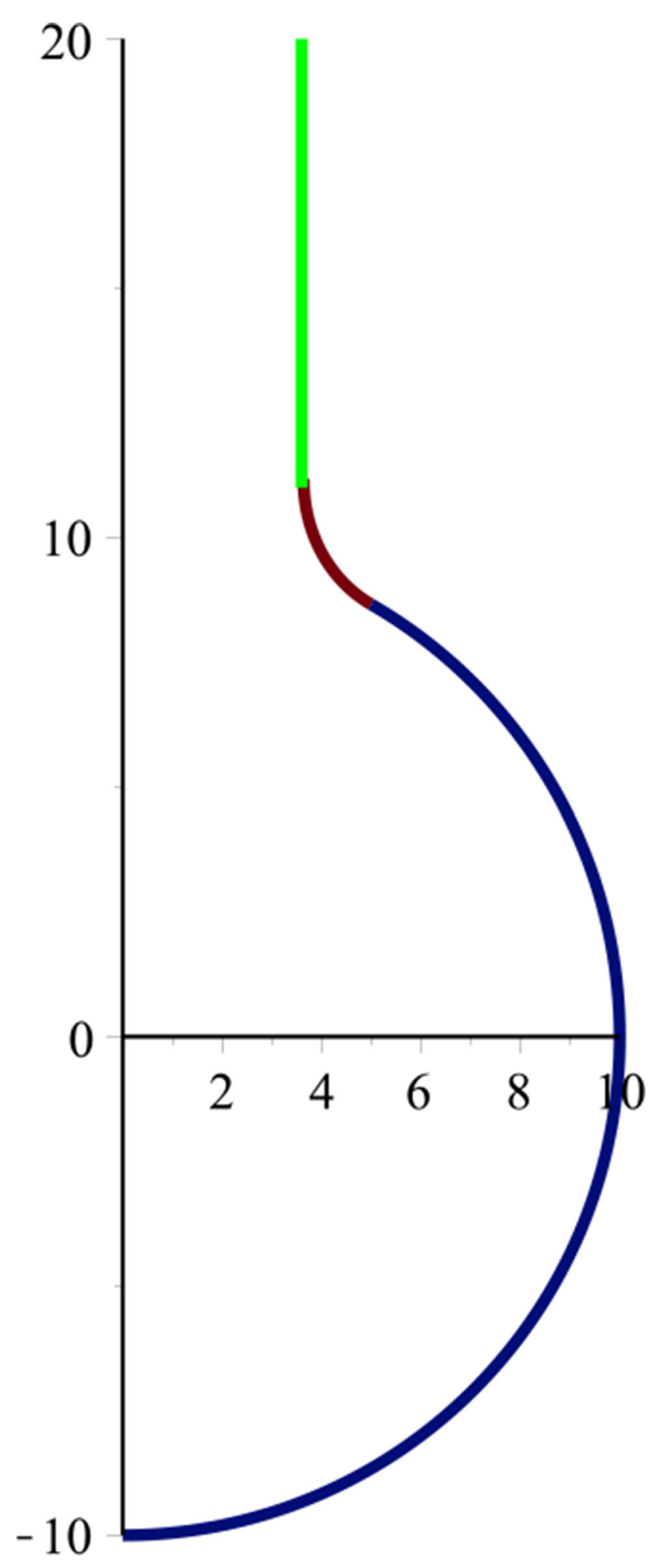

Fig. 3 Connection profile between a BN nanotube and fullerene based on the Willmore energy

As a result, we find $A_{1}$ and $A_{2}$ to be

$A_{1}=r_{t} \cosh ^{-1}\left(\sqrt{\frac{R_{1}}{r_{t}}}\right)+\sqrt{R_{1}\left(R_{1}-r_{t}\right)}$,

\section{BN Fullerene}

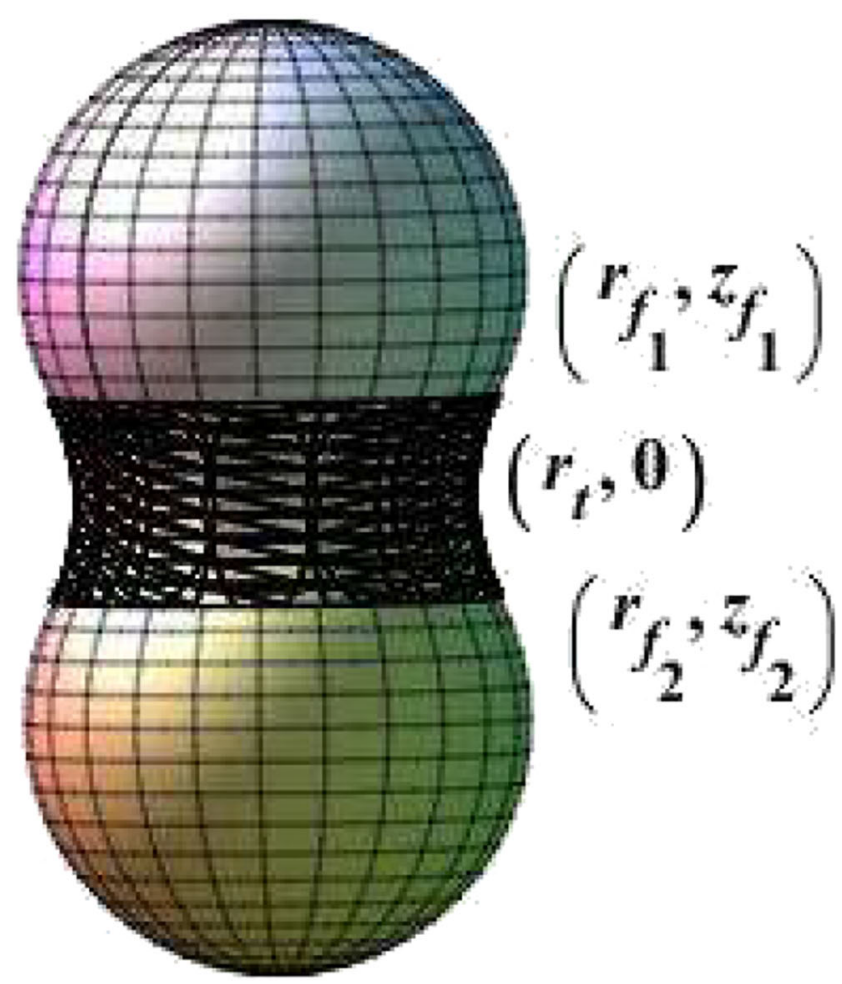

\section{BN Fullerene}

Fig. 4 A part of the catenoid joins between two BN fullerenes

$A_{2}=-r_{t} \cosh ^{-1}\left(\sqrt{\frac{R_{2}}{r_{t}}}\right)+\sqrt{R_{2}\left(R_{2}-r_{t}\right)}$.

By substituting Eqs. 7, 12, and 13 into Eqs. 6, 8, and 9 with specified values of $r_{t}$ and $R_{1}=R_{2}$, we obtain the connection shape between two fullerenes by using the catenoid curve, as shown in Fig. 5.

\section{Joining a BN fullerene and BN nanotorus}

In this case, we seek to join two different BN nanostructures, a fullerene and a torus, by using the Willmore energy, as shown in Fig. 6. We can divide this case into two parts: upper and lower parts. First, the upper part of the catenoid will join a fullerene. This case is similar to the upper part of the previous "Joining two BN fullerenes." Therefore, we have the same calculation as this integration, and we consider Eq. 12 on this side. Second, the lower part of 


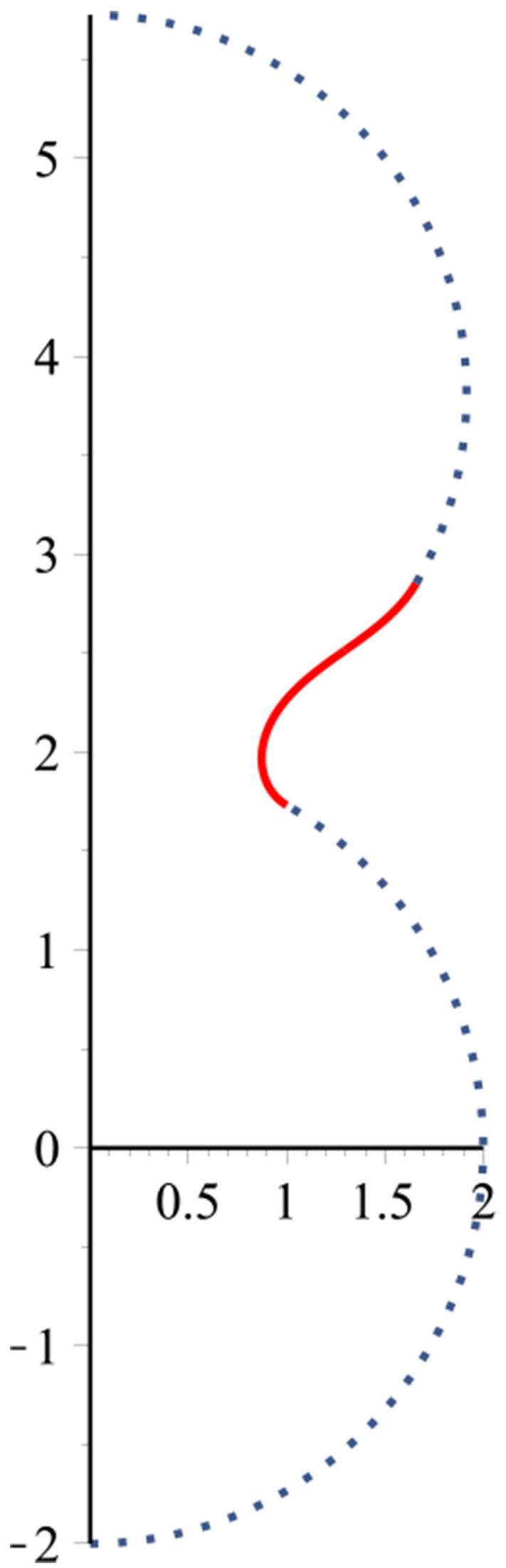

Fig. 5 Connection profile of two BN fullerenes using the Willmore energy

\section{BN Fullerene}

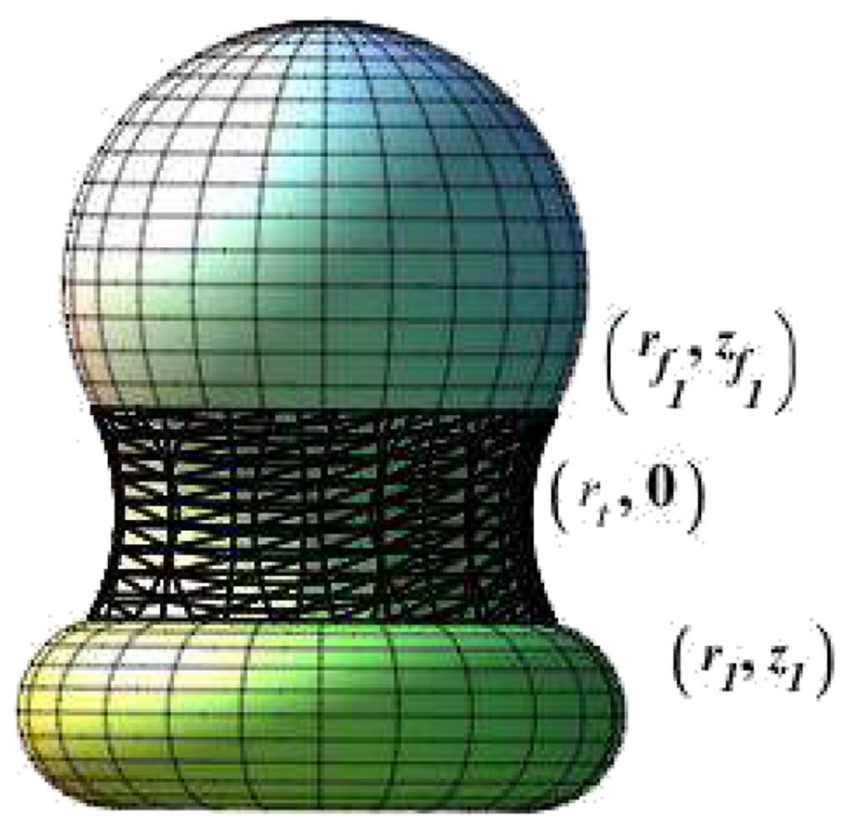

\section{BN nanotorus}

Fig. 6 A part of the catenoid joins between a BN fullerene and nanotorus

the catenoid will join a torus. The equation of a torus in Cartesian coordinates is

$\left(\sqrt{x^{2}+y^{2}}-R\right)^{2}+z^{2}=a^{2}$,

and after a transformation, we obtain

$x(\phi, \theta)=(R+a \cos \theta), \cos \theta$

$y(\phi, \theta)=(R+a \cos \theta), \sin \theta$

$z(\phi, \theta)=a \sin \theta$,

where $\theta$ and $\phi$ are polar and azimuthal angles in the $x$ axis and $x y$-plane, respectively. Additionally, $R$ denotes the major radius of the torus, and $a$ denotes the minor radius of the torus. Thus, the equation of the torus in cylindrical coordinates is $(r-R)^{2}+z^{2}=a^{2}$, which can be written as

$z=D+\sqrt{a^{2}-(r-R)^{2}}$,

where $D$ is a constant that can be determined by the position of the torus along the negative $z$-axis [30]. Now, at the joining point $\left(r_{1}, z_{1}\right)$, considering the negative sign of Eq. 6 , which represents the lower part of the catenoid, and Eq. 7 along with Eq. 14, we obtain

$$
\begin{aligned}
-\frac{\cosh ^{-1}\left(a r_{1}\right)}{a} & =D+\sqrt{a_{1}^{2}-\left(r_{1}-R\right)^{2}}, \\
D & =-r_{t} \cosh ^{-1}\left(\frac{r_{1}}{r_{t}}\right)-\sqrt{a_{1}^{2}-\left(r_{1}-R\right)^{2}} .
\end{aligned}
$$


Next, if we match the gradient, we have

$$
-\frac{1}{\sqrt{a^{2} r_{1}^{2}-1}}=\frac{r_{1}-R}{\sqrt{a_{1}^{2}-\left(r_{1}-R\right)^{2}}},
$$

then, by substituting Eq. 7, we obtain

$r_{1}=\frac{\sqrt{4 a_{1} r_{t}+R}+R}{2}$

which results in

$$
\begin{aligned}
D= & -r_{t} \cosh ^{-1} \frac{\sqrt{4 a_{1} r_{t}+R}+R}{2 r_{t}} \\
& -\sqrt{a_{1}^{2}-\left(\frac{\sqrt{4 a_{1} r_{t}+R}-R}{2}\right)^{2}} .
\end{aligned}
$$

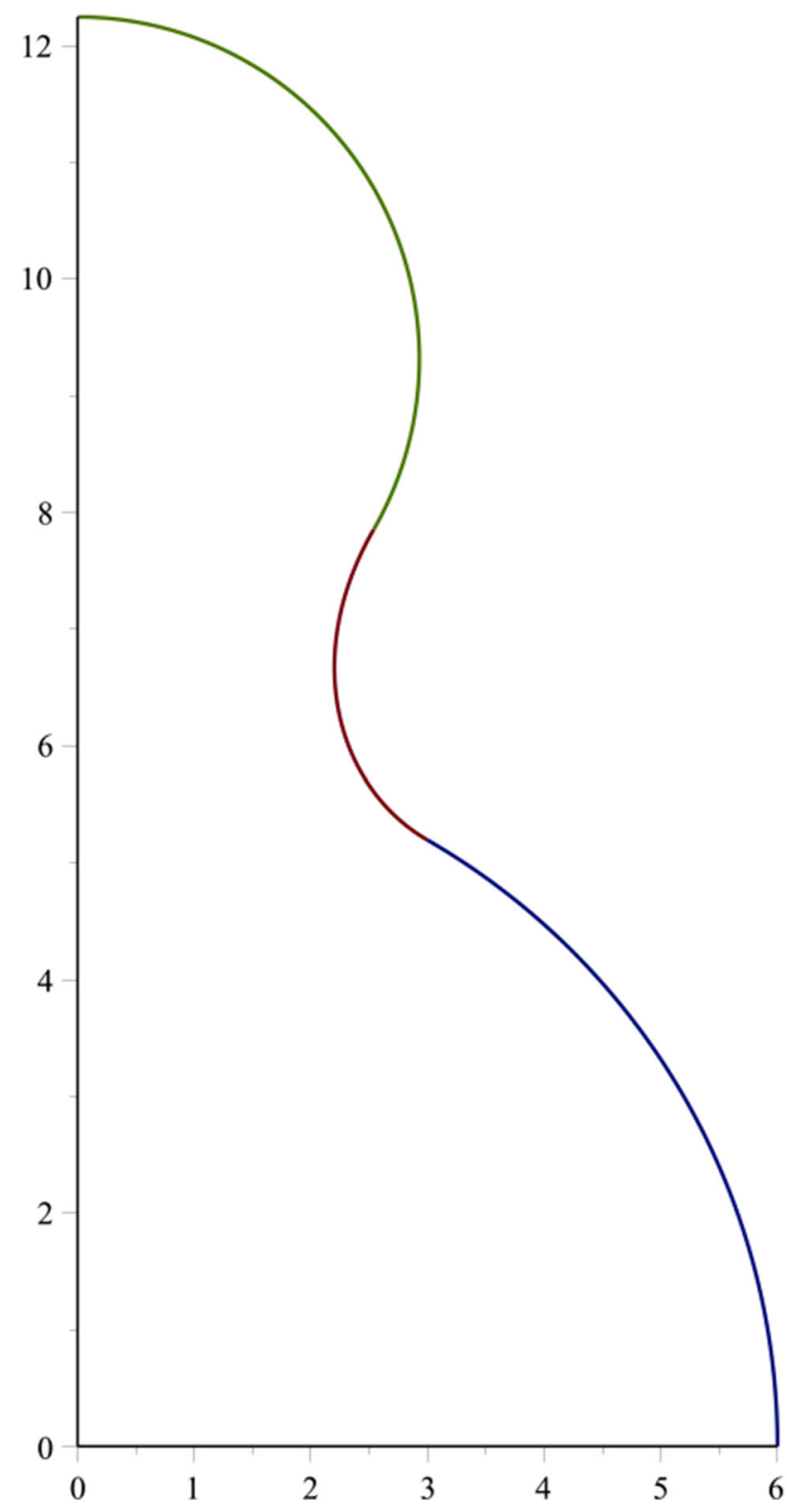

Fig. 7 Connection profile of a $\mathrm{BN}$ fullerene and nanotorus by using the Willmore energy
Now, by substituting Eqs. 7, 12 and 15 into Eqs. 6, 8 and 14, respectively, with specified values of $r_{t}, R_{1}, a_{1}$, and $R$, we obtain the joining shape between the fullerene and torus by using the catenoid curve, as shown in Fig. 7.

\section{Conclusions}

In conclusion, calculus of variations is utilized to determine the connection scenarios among boron nitride nanostructures. Minimizing the elastic energy to define the connection area between carbon nanostructures has been used in many studies, while the problem is considered to be in a twodimensional $x y$-plane. In addition, this method has been applied in the literature to join boron nitride nanostructures because of their similarity to carbon nanostructures. Furthermore, minimizing the Willmore energy, which is an extension of the elastic energy, can specify the joining curve between carbon nanostructures, while in this case, this problem is treated as a three-dimensional problem. In particular, a part of the catenoid has been used in this setting because it is a minimal energy surface. In this research, the Willmore energy minimization method is extended to cover the joining scenarios of other nanomaterials, that is, boron nitride structures at the nanoscale. The results of this method are provided for three different joining structures, namely, a $\mathrm{BN}$ nanotube and $\mathrm{BN}$ fullerene, a $\mathrm{BN}$ fullerene and $\mathrm{BN}$ fullerene, and a $\mathrm{BN}$ fullerene and $\mathrm{BN}$ nanotorus. Note that all the shapes used are assumed to be geometrically perfect structures and have not been deformed from the original shapes. The main aim in this study is to formulate the underlying axially symmetric model so that we have a reference basis for the comparison of real physical structures. Although there are no experimental or computational results for comparison, simple models appear to give rise to meaningful approximations to complex structures and therefore might be useful for future work on this problem. Ultimately, various nanoscale $\mathrm{BN}$ structures might be connected by considering the same method, which may be considered future work.

Author contribution N.A. wrote the main manuscript text, prepared all figures, and reviewed the manuscript.

Funding The author did not receive support from any organization for the submitted work.

Availability of data and material N/A

Code availability N/A

\section{Declarations}

Ethics approval N/A 


\section{Consent to participate N/A}

\section{Consent for publication N/A}

Conflict of interest The author declares no competing interests.

Open Access This article is licensed under a Creative Commons Attribution 4.0 International License, which permits use, sharing, adaptation, distribution and reproduction in any medium or format, as long as you give appropriate credit to the original author(s) and the source, provide a link to the Creative Commons licence, and indicate if changes were made. The images or other third party material in this article are included in the article's Creative Commons licence, unless indicated otherwise in a credit line to the material. If material is not included in the article's Creative Commons licence and your intended use is not permitted by statutory regulation or exceeds the permitted use, you will need to obtain permission directly from the copyright holder. To view a copy of this licence, visit http://creativecommons. org/licenses/by/4.0/.

\section{References}

1. Alshammari N, Thamwattana N, McCoy J, Duangkamon B, Cox B, Hill J (2018) Modelling joining of various carbon nanostructures using calculus of variations. Dynamics of Continuous, Discrete and Impulsive Systems Series B: Applications and Algorithms 25:307-339

2. Alshammari N, Thamwattana N, McCoy J, Duangkamon B, Cox B, Hill J (2018) Modelling joining of various carbon nanostructures using calculus of variations. Dynamics of Continuous, Discrete and Impulsive Systems Series B: Applications and Algorithms 25:307-339

3. Alshammari N (2020) Joining between boron nitride nanocones and nanotubes. Advances in Mathematical Physics 2020:1-6

4. Alshammari N (2020) Mathematical modelling for joining boron nitride graphene with other bn nanostructures. Advances in Mathematical Physics

5. Bozic B, Das S, Svetina S (2015) Sorting of integral membrane proteins mediated by curvature-dependent protein-lipid bilayer interaction. Soft Matter 11:2479-2487

6. Carvalho-Santos D (2012) Coupling between magnetic field and curvature in heisenberg spins on surfaces with rotational symmetry. Phys Lett A 376:3551-3554

7. Carvalho-Santos V, Apolonio F, Oliveira-Neto N (2013) On geometry-dependent vortex stability and topological spin excitations on curved surfaces with cylindrical symmetry. Phys Lett A 377:1308-1316

8. Chee L, Bhandari S, Tiwari B, Yapici N, Zhang D, Yap Y (2016) Boron nitride nanotubes: Recent advances in their synthesis, functionalization, and applications. Molecules 21(7):922

9. Cox B, Hill J (2008) A variational approach to the perpendicular joining of nanotubes to plane sheets. Journal of Physics A: Mathematical and Theoretical 41:1-2

10. Dai Y, Jiang H, Hu Y, Li C (2013) Hydrothermal synthesis of hollow mn2o3 nanocones as anode material for li-ion batteries. RSC Adv 3:19778-19781
11. Duangkamon B, Cox B, Hill J (2013) Determination of join regions between carbon nanostructures using variational calculus. ANZIAM J 54:221-247

12. Genoese A, Genoese A, Salerno G (2020) Hexagonal boron nitride nanostructures: a nanoscale mechanical modeling. J Mech Mater Struct 15:249-275

13. Golberg D, Bando Y, Huang Y et al (2010) Boron nitride nanotubes and nanosheets. ACS Nano 4(6):2979-2993

14. Golberg D, Bando Y, Tang C, Zni C (2007) Boron nitride nanotubes. Adv Mater 19(18):2413-2432

15. Hao Y, Wang R, Li C (2012) Minimal quasi-bézier surface. Appl Math Model 36:5751-5757

16. Kim S, Khai T, Kwon Y et al (2015) One-pot synthesis of hbn fullerenes usinsg a graphene oxide template. Met Mater Int 21:950-955

17. Koi N, Oku T, Nishijima M (2005) Fe nanowire encapsulated in boron nitride nanotubes. Solid State Commun 136(6):342-345

18. Loh G, Baillargeat D (2014) Thermal transport in boron nitride nanotorus-towards a nanoscopic thermal shield. J Appl Phys 114:183502

19. Mackay D, Janish M, Sahaym U, Kotula P, Jungjohann K, Carter C, Norton M (2014) Template-free electrochemical synthesis of tin nanostructures. J Mater Sci 49:1476-1483

20. Man W, Chang C, Zettl A (2004) Encapsulation of onedimensional potassium halide crystals within bn nanotubes. Nano Lett 4(7):1355-1357

21. Marin M, Ellahi R, Chirila A (2017) On solutions of saint-venant's problem for elastic dipolar bodies with voids. Carpathian Journal of Mathematics 33:219-232

22. Marin M, Nicaise S (2016) Existence and stability results for thermoelastic dipolar bodies with double porosity. Continuum Mech Thermodyn 28:1645-1657

23. Mickelson W, Aloni S, Han W, Cumings J, Zettl A (2003) Packing c60 in boron nitride nanotubes. Science 300(5618):467-469

24. Nawa A Mathematical modelling in nanotechnology using calculus of variations. Doctor of philosophy thesis, School of Mathematics and Applied Statistics, University of Wollongong

25. Ramon S (2015) Ph.D. thesis, University of San Luis Potosi

26. Riviere T (2008) Analysis aspects of willmore surfaces. Invent Math 174:1-45

27. Rouhi S, Ansari R, Shahnazari A (2016) Vibrational characteristics of single-layered boron nitride nanosheet/single-walled boron nitride nanotube junctions using finite element modeling. Materials Research Express 3:125027

28. Scida K, Stege P, Haby G, Messina G, Garcia C (2011) Recent applications of carbon-based nanomaterials in analytical chemistry: Critical review. Anal Chim Acta 691:6-17

29. Sripaturad P, Alshammari P, Thamwattana N, McCoy J, Duangkamon D (2018) Willmore energy for joining of carbon nanostructures. Phil Mag 98:1511-1524

30. Sripaturad P, Baowan D (2021) Joining curves between nanotorus and nanotube: mathematical approaches based on energy minimization. Z Angew Math Phys 72:2-11

31. Willmore T (1965) Note on embedded surfaces. An St Univ Iasi Mat 12B:493-496

32. Yec C, Zeng H (2014) Synthesis of complex nanomaterials via ostwald ripening. J Mater Chem A 2:4843-4851

Publisher's note Springer Nature remains neutral with regard to jurisdictional claims in published maps and institutional affiliations. 